\title{
A New Microfluidic Phase-Transfer Reaction Using HPLC Guard Columns as the Reactor for the $\mathrm{N}^{3}$-Protection of Uridine Deriva- tives
}

Nobuhiro Tago

Yoshiaki Masaki

Hiroshi Nagasawa

Takashi Kanamori

Akihiro Ohkubo

Kohji Seio*

Mitsuo Sekine*

Synlett 2015, 26, 2578.

For the final online and print versions of this article, tree branch was replaced by branch unit on the second page, left column, line 7. The editorial office apologizes profusely for overlooking this correction for the advance online publication (e-First) version of this article. 